\title{
Staged hybrid ablation for persistent and longstanding persistent atrial fibrillation effectively restores sinus rhythm in long-term observation
}

Michal Zembala, Krzysztof Filipiak, Oskar Kowalski, Piotr Buchta, Tomasz Niklewski, Pawel Nadziakiewicz, Rafał Koba, Mariusz Gąsior, Zbigniew Kalarus, Marian Zembala

Department of Cardiac Surgery and Transplantology, Silesian Center For Heart Diseases, Zabrze, Poland

Submitted: 12 May 2015

Accepted: 3 July 2015

Arch Med Sci 2017; 13, 1: 109-117

DOI: $10.5114 /$ aoms.2015.53960

Copyright $\odot 2016$ Termedia \& Banach

\section{Abstract}

Introduction: Hybrid ablation (HABL) of atrial fibrillation combining endoscopic, minimally invasive, closed chest epicardial ablation with endocardial CARTO-guided accuracy was introduced to overcome the limitations of current therapeutic options for patients with persistent (PSAF) and longstanding persistent atrial fibrillation (LSPAF). The purpose of this study was to evaluate the procedural safety and feasibility as well as effectiveness of HABL in patients with PSAF and LSPAF 1 year after the procedure.

Material and methods: The study is a single-center, prospective clinical registry. From 07/2009 to $12.2014,90$ patients with PSAF $(n=39)$ and LSPAF $(n=51)$, at the mean age of $54.8 \pm 9.8$, in mean EHRA class 2.6 , underwent HABL. $64.4 \%$ of patients had a history of prior cardioversion or catheter ablation. Thirteen patients had LVEF less than $35 \%$. Mean AF duration was $4.5 \pm 3.7$ years. Patients were scheduled for 3-, 6- and 12-month follow-up with 7-day Holter monitoring.

Results: At 6 months after the procedure 78\% (54/69) of patients were in SR. At 12 months after the procedure $86 \%(59 / 69)$ were in SR and $62.3 \%$ (43/69) in SR and off class I/III antiarrhythmic drugs (AADs). Only $1 \%(1 / 69)$ of patients required a repeat ablation for atrial flutter. A significant decrease in LA dimension and an increase in LVEF were noted.

Conclusions: A combination of epicardial and endocardial RF ablation should be considered as a treatment option for patients with persistent and long-standing persistent atrial fibrillation as it is safe and effective in restoring sinus rhythm.

Key words: atrial fibrillation, ablation, hybrid procedures, pulmonary vein isolation.

\section{Introduction}

Atrial fibrillation (AF) is the most common cardiac arrhythmia, affecting nearly $2 \%$ of the general population worldwide. It is associated with an increased risk of stroke and is an independent risk factor for sudden death $[1,2]$. While pharmacologic management remains the first line of therapy for AF, its effectiveness sharply declines as the disease progresses. With the paucity of innovations, patients are bound long-term to antiarrhythmic drugs (AADs), as novel substances have failed to prove their superiority. Catheter ablation, which primarily aims at pulmonary vein

\author{
Corresponding author: \\ Michal Zembala MD, PhD \\ Department of Cardiac \\ Surgery and Transplantology \\ Silesian Center For \\ Heart Diseases \\ 9 Sklodowskiej St \\ 41-800 Zabrze, Poland \\ Phone: +48600402994 \\ E-mail: m@sccs.pl
}


isolation, is well established, safe and effective in termination of paroxysmal AF, but lacks efficacy in persistent and especially in long-standing persistent AF; even with repeat procedures, long-term success rates of less than $50 \%$ have been reported $[3,4]$. On the other hand, surgical standalone procedures, based on the Cox-Maze concept, despite their progression to less invasive procedures and positive results, remain under-utilized. Recently updated guidelines still limit surgical ablation to failed percutaneous attempts in highly symptomatic patients, not indicating, however, the number of percutaneous attempts for these individuals. Regardless of a significant gap in reported evidence, a minimally invasive surgical procedure is rarely proposed in long-suffering AF patients, even after failing one or more catheter ablations, as many of these patients are still considered to be candidates for percutaneous intervention.

Hybrid ablation of atrial fibrillation ( $H A B L$ ) is a combination of endocardial and epicardial ablation, designed to overcome the challenges of individual surgical and catheter procedures [5]. While minimally invasive, totally endoscopic surgical technique utilizes a unique transabdominal access to the pericardium allowing for unrestricted ablation of the posterior left atrium, CARTO-guided endocardial ablation is used to close isolation lines, complete circumferential pulmonary vein lines and test entry and exit blocks [6]. This single-center, all-comers clinical registry documents

Table I. Patient demographics and baseline characteristics

\begin{tabular}{|lc|}
\hline Baseline characteristics & Average \pm SD (range) \\
\hline Age [years] & $54.8 \pm 9.8(28-75)$ \\
\hline AF duration [years] & $4.5 \pm 3.7(1-20)$ \\
\hline Left atrial size [cm] & $4.5 \pm 0.6(3.2-5.9)$ \\
\hline LVEF (\%) & $48.6 \pm 9.7(20-65)$ \\
\hline Body mass index [kg/m²] & $28.9 \pm 3.7(22-42)$ \\
\hline CHA DS VASc & $1.7 \pm 1.2(0-6)$ \\
\hline EHRA & $2.6 \pm 0.6(2-4)$ \\
\hline NYHA & $2.0 \pm 0.5(0-3)$ \\
\hline Baseline characteristics & Percentage (\%) \\
\hline Gender & $78 \%$ male; \\
\hline$\%$ Paroxysmal AF & $22 \%$ female \\
\hline$\%$ Persistent AF & $0 \%(0 / 90)$ \\
\hline$\%$ Longstanding persistent AF & $43 \%(39 / 90)$ \\
\hline $\begin{array}{l}\text { alone } \\
\text { alone }\end{array}$ & $20.0 \%(18 / 90)$ \\
\hline cardioversion & $21.1 \%(19 / 90)$ \\
\hline
\end{tabular}

one-year safety and efficacy outcomes of the treatment of symptomatic patients with non-paroxysmal atrial fibrillation.

\section{Material and methods}

The appropriate Institutional Review Board (IRB) and Ethics Committee approvals were obtained for the study prior to initiating patient enrolment. From July 2009 to December 2014, a total of 90 patients underwent HABL. Thirty-nine (43\%) were diagnosed with persistent AF (PSAF) and 51 (57\%) with long-standing persistent AF (LSPAF). The 90 patients enrolled in the study had a mean age of $54.8 \pm 9.80$ years (range: $28-75$ years) and $78 \%$ (43/55) were male. Mean AF duration was $4.5 \pm 3.7$ years (range: $1-20$ years) and the mean European Heart Rhythm Association (EHRA) class was 2.6. In $64.4 \%(58 / 90)$ of patients, AF treatment, restoration of SR, was attempted either by electrical cardioversion only $(21.1 \%, 19 / 90)$, endocardial catheter ablation only $(20.0 \%, 18 / 90)$, or both endocardial catheter ablation and electrical cardioversion $(23.3 \%, 21 / 90)$ prior to the HABL. Of the 39 patients who had previous catheter ablation, $56.4 \%(22 / 39)$ of patients had two or more catheter ablations with a mean of 2 procedures per patient (max. 4, min. 1). Failure of percutaneous treatment was defined as recurrence of AF lasting over $48 \mathrm{~h}$ (documented) or requiring cardioversion after a 3-month blanking period. Patients with a history of complicated percutaneous pulmonary vein isolation procedure (tamponade, perforation, pulmonary vein stenosis $>50 \%$ ) were not eligible to undergo the HABL procedure. Tachyarrhythmic cardiomyopathy, defined as left ventricular ejection fraction (LVEF) equal to or less than 35\% due to long-lasting arrhythmia, was diagnosed in $14.4 \%(13 / 90)$ of patients. Hypertensive heart disease was present in $66.8 \%(62 / 90)$ of patients and diabetes mellitus in $13.3 \%$ (12/90) of patients. Amiodarone-induced hyperthyroidism was present in $14.4 \%(13 / 90)$ of patients. Pulmonary anomalies were observed in $26.7 \%$ (24/90) of patients.

Table I summarizes patient demographics and baseline clinical characteristics.

The study was designed as a single-center, prospective, non-randomized clinical registry. A Heart Team consisting a cardiac surgeon and electrophysiologist screened consecutive patients with drug-refractory PSAF or LSPAF for study eligibility. Adult patients over 18 years of age, willing to comply with the required follow-up and providing their informed consent were enrolled. Patients having paroxysmal $A F$, any previous cardiac surgery, or who had not failed a previous class I/III antiarrhythmic drug (AADs) pharmacological therapy were subject to exclusion from study participation. 
The aim of the study was to document both safety and effectiveness of the HABL 1 year after the initial treatment as well as its feasibility. Safety was defined as occurrence of major adverse cardiac events such as death, myocardial infarction, stroke, major bleeding or any procedure-related life-threatening event that occurred during the procedure or throughout the 12-month period following the procedure. Effectiveness was defined according to the HRS/EHRA/ECAS consensus as freedom from AF measured by lack of atrial tachyarrhythmia > $30 \mathrm{~s}$ as documented by 7-day Holter monitoring at 12 months after the procedure [7]. Additional evaluated endpoints included sinus rhythm (SR) off AADs and sinus rhythm (SR) without any intervention (e.g. cardioversion or repeat ablation). Feasibility was defined by the percentage of patients who completed $\mathrm{HABL}$ according to the study protocol and the percentage of procedures requiring conversion to a sternotomy. Detailed study protocols, surgical and percutaneous CARTO procedures and preliminary results were published previously $[8,9]$. Patients were anesthetized using the total intravenous anesthesia (TIVA) technique together with short acting muscle relaxants. Two independent temperature probes were inserted into the esophagus. A midline abdominal $2 \mathrm{~cm}$ incision was made $3 \mathrm{~cm}$ below the xyphoid through which a $10 \mathrm{~mm}$ laparoscopic port was inserted. Once the peritoneum was accessed $\mathrm{CO}_{2}$ insufflation was initiated and two $5 \mathrm{~mm}$ working ports were inserted in the left and right subcostal area. The central tendon of the diaphragm was identified and incised

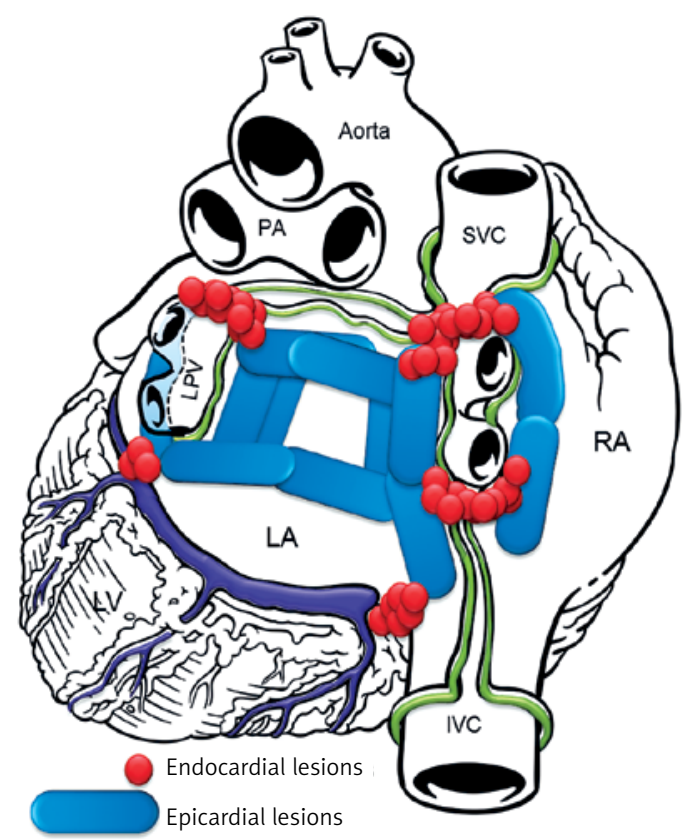

Figure 2. Lesion pattern a created mostly in PSAF patients

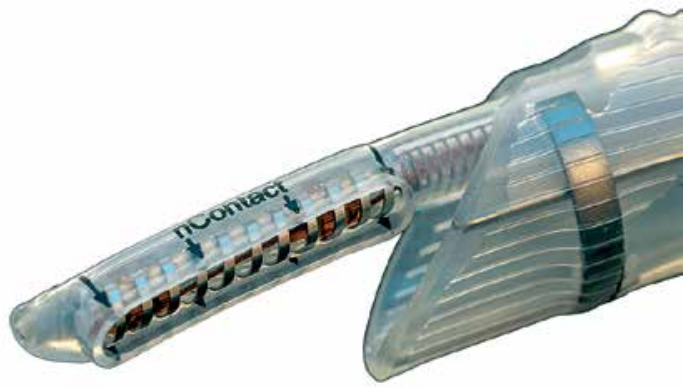

Figure 1. Radiofrequency epicardial ablation device in the dedicated cannula

horizontally $(3-4 \mathrm{~cm})$ with a harmonic scalpel (Ethicon, USA). The pericardium was subsequently entered and the laparoscopic ports removed. A cannula designed for pericardioscopic access (nContact Surgical, Morrisville, NC, USA - Figure 1) was then placed inside the pericardial sac. The cannula with an endoscope was pushed into the oblique sinus to visualize the posterior wall of the left atrium (LA) and pulmonary veins (PVs). An irrigated, unipolar radiofrequency ablation device (VisiTrax nContact Surgical, Morrisville, NC, USA) was passed through the cannula. Linear lesions (90 s each, $30 \mathrm{~W}$ power) were created. Due to the evolution of the surgical technique and our understanding of the AF pathology (LA fibrosis), the lesion pattern evolved over time. Patients with PSAF were more likely to receive pattern $A$ (Figure 2), while patients with LSPAF and with a history of endocardial ablations were treated more aggressively with lesion pattern B (Figure 3). All applica-

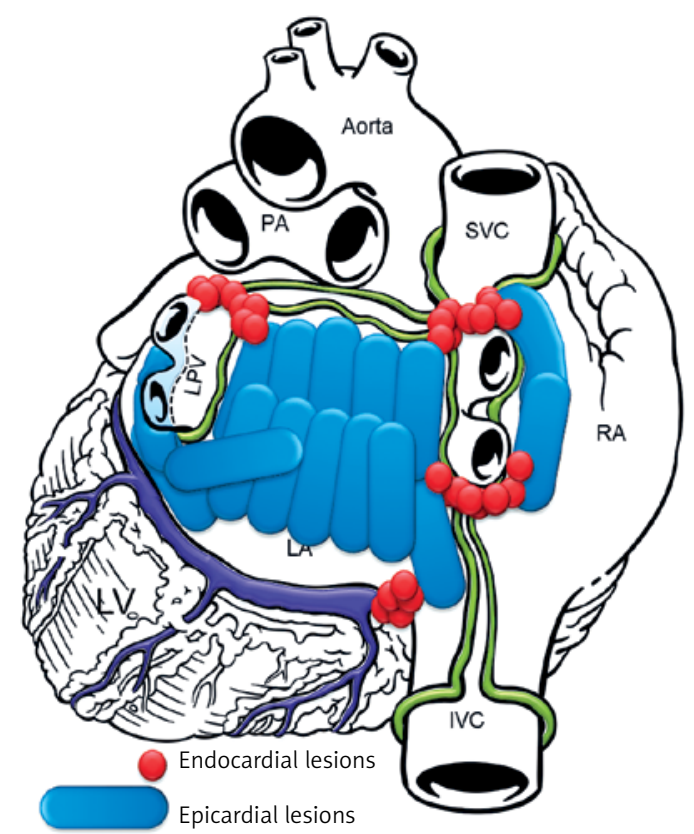

Figure 3. Lesion pattern B created mostly in LSPAF patients 
tions on the posterior wall of the left atrium were performed under fluoroscopic guidance to visualize the relation between the ablating electrode and the esophagus. Temperature probes were positioned to match the position of the ablating electrode. $50 \mathrm{ml}$ of $30^{\circ} \mathrm{C} 0.9 \%$ saline was injected into the pericardium prior to RF energy application to submerge the ablating electrode and reduce temperature spread. When all lesions had been created, a small drain was placed behind the LA and passed through one of the $5 \mathrm{~mm}$ endoscopic ports. The midline fascia was closed with interrupted permanent sutures. Skin and port incisions were closed using absorbable sutures. Endocardial ablation procedures were performed using the Seldinger technique to introduce two sheaths via peripheral veins. A 10-polar $6 \mathrm{~F}$ electrode, which served as the reference, was introduced into the coronary sinus via the right internal jugular vein. Subsequently, the Brockenbrough needle and the Mullins-type transseptal sheath were both positioned in the upper right atrium and the transseptal puncture was performed under the guidance of intracardiac pressures recorded from the tip of the needle. Immediately after the puncture, a single bolus of 10,000 U of heparin was administered, and an 8F NaviStar irrigated-tip ablation electrode (Biosense Webster, Diamond Bar, CA, USA) was introduced into the left atrium. Using an electro-anatomical mapping system (CARTO, Biosense Webster) the isopotential map of the left atrium was created to identify areas showing electrical activity within the pulmonary vein ostia and in the region of the left atrial isthmus. Electrical silence was indicated if the amplitudes of bipolar atrial electrograms were $<0.05 \mathrm{mV}$. Once identified and marked on the map, areas with persistent conductivity were subsequently ablated using radiofrequency applications limiting the power to $40 \mathrm{~W}$ and application time to $60 \mathrm{~s}$. Finally, the electrical isolation of the veins was verified by stimulating the pulmonary ostia with a cycle length of 500 ms (Figure 4).

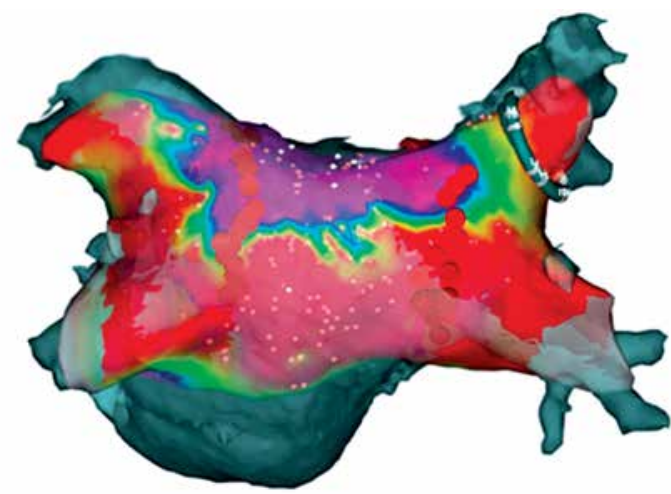

Figure 4. CARTO mapping of the left atrium after the convergent procedure

\section{Anticoagulation protocol and changes in $A A D$ regimen}

All patients were prescribed the same postoperative pharmacological protocol: intravenous infusion of amiodarone (Cordarone, $600 \mathrm{mg} / 50 \mathrm{ml}$ $2 \mathrm{ml} / \mathrm{h}$ ) together with IV heparin (25000 IU/50 ml $2 \mathrm{ml} / \mathrm{h} ;$ ACT $\approx 180 \mathrm{~s}$ ) was initiated $1 \mathrm{~h}$ after surgery and continued for $48 \mathrm{~h}$. Potassium was supplemented to achieve levels in the range of 4.5-4.7. Warfarin (VKA) treatment was initiated on postoperative day two to maintain the international normalized ratio (INR) at 2.5-3.5 in all patients who underwent the HABL in a single setting $(n=8)$. Patients who underwent the staged procedure were discharged either on low molecular weight heparin (LMWH Clexane, $1 \mathrm{mg} / \mathrm{kg}$ twice daily) or the novel oral anticoagulant (NOAC) rivaroxaban, Xarelto, Bayer, $1 \times 20 \mathrm{mg}(n=14)$, and were continued on their prescribed therapy until readmission for the endocardial ablation portion of the procedure. Upon completion of stage II, patients were discharged either on VKA or NOAC, depending on the preoperative regimen or patient preferences. For the first 3 months, patients were prescribed the same AADs as prior to $\mathrm{HABL}$. Changes in AAD therapy were made carefully once the 3-month blanking period expired, with a reduction or discontinuation of $A A D$ therapy over the next 6-12 months as medically indicated. Aspirin (75 mg) was substituted for warfarin at 6 months after the procedure if maintenance of sinus rhythm was confirmed, unless there were other indications for systemic anticoagulation such as $\mathrm{CHADS}_{2} \geq 2[10,11]$. In patients with confirmed sinus rhythm and low risk of thromboembolic events (defined as CHA2DS2-VASC 0) anticoagulation therapy was discontinued and substituted for $1 \times 75 \mathrm{mg}$ aspirin (ASA). In patients with a CHA2DS2-VASc score of 1 and HAS-BLED > 3, VKA or NOACs were also suggested to be discontinued and replaced with ASA, due to increased risk of bleeding. When the CHA2DS2-VASc score was 1 and HAS-BLED < 3, oral anticoagulation was advocated. However, in all patients with CHA2DS2-VASc of 2 and greater, anticoagulation, despite sinus rhythm, was not recommended.

\section{Monitoring and data collection}

All patients were seen in an outpatient clinic at 6 and 12 months after the procedure (patients with implanted REVEAL XT were monitored every 3 months after the procedure). Five patients had ECG Loop Monitors (Reveal XT) implanted at the time of the epicardial ablation procedure. For the remaining patients, $24 \mathrm{~h}$ Holter monitoring was performed at 3 months and seven-day Holter monitoring at 6- and 12-month visits. Transtho- 
racic echocardiography (TTE) was performed 6 and 12 months postoperatively.

\section{Statistical analysis}

Continuous variables are expressed as mean \pm 1 SD. Continuous parameters were compared with the Student $t$ test. A $p$-value $\leq 0.05$ indicates statistical significance. The authors had full access to and take full responsibility for the integrity of the data. All authors have read and agreed to the manuscript as written.

\section{Results}

\section{Safety data analysis}

A total of 4 serious adverse events (SAEs) were reported throughout the study duration: death of unknown cause, bleeding requiring sternotomy, cardiac tamponade and transient ischemic attack (TIA). These events have been described in detail and discussed in our preliminary report [8]. Since the original publication, one serious (cardiac tamponade), and three minor, reversible conditions (a transient ischemic attack (TIA), pericardial effusion and temporary phrenic nerve palsy) were encountered.

Clinical signs of cardiac tamponade were noticed shortly after the endocardial procedure (stage II). The condition was treated in the operating room with placement of a drain in the pericardial cavity via subxyphoid incision, as no sternal incision was required. A careful "watch-and-wait" strategy was adopted, until the patient was hemodynamically stable. Three days later the drain was removed, and the patient had an uneventful recovery.

In the case of pericardial effusion, the patient experienced signs of inflammation shortly after the epicardial ablation, possibly due to the extensive ablation of the posterior atrial wall required due to the fibrosis and size of the left atrium. The condition was managed with steroids, as the amount of pericardial fluid did not restrict myocardial wall movement, resolving within 4 weeks. As the patient remained in stable SR throughout the entire treatment, he subsequently refused to undergo the endocardial component of the staged HABL. Phrenic nerve palsy occurred in a patient with difficult right side anatomy, with an additional pulmonary vein. While unnoticed by the patient, the higher position of the right diaphragm on the chest X-ray and its limited contractility on cine confirmed the diagnosis. The changes resolved within 3 months, as the patient was followed up closely. Lastly, transient ischemic attack was noted in a patient with a history of stroke, 2 days after an epicardial procedure. The event was limited to parietal loss of vision, and occurred during tran- sition from LMWH to rivaroxaban (Xarelto, Bayer) and resolved quickly without neurological deficit.

Importantly, SAEs typical and distinct for surgical and percutaneous procedures could be observed, magnifying the significance of staging of the two procedures.

\section{Feasibility observations}

The procedure was feasible in all but one patient, as the individual had a history of gastric ulcer perforation and subsequent peritonitis, treated surgically in 2001. An attempt was made to enter the peritoneal cavity, with lateral placement of endoscopic ports to avoid the previous incision line. Despite careful maneuvers aiming at dissection of severe peritoneal adhesions, the procedure was discontinued to mitigate the risks of extensive dissections. Although our and other experience shows that pericardioscopy can be safely performed in patients with a history of multiple abdominal procedures, this individual had severe adhesions, which prevented safe access to the diaphragm.

As previously reported, the HABL was performed as a staged procedure to accommodate the local reimbursement challenges that created a need for stable anticoagulation therapy during intermission. While most of the patients tolerated this inconvenience well, 1 patient despite LMWH had blood stasis and "spontaneous" echo contrast noted in the left atrial appendage (LAA) prior to stage II (endocardial ablation), and the endocardial ablation was delayed. Once the LAA proved clear, the patient underwent successful LAA exclusion with the Watchman device, followed by the CARTO guided endocardial ablation completing the protocol.

$37.8 \%$ (34/90) of patients had the study-defined lesion pattern A, while in $62.2 \%(56 / 90)$ of patients lesion pattern B was created. Conversion from AF to SR during the procedure was noted in $19 \%(17 / 90)$ of patients, while the remaining $81 \%$ (73/90) underwent successful electrical cardioversion in the OR shortly after all epicardial applications had been completed. In 2 patients the LAA was removed, via the left thoracoscopic approach. In these cases the LAA was removed first with an endoscopic stapler (GI Ethicon) followed by creation of a roof line and coagulation of the ligament of Marshall. The surgical procedure took on average $113 \pm 23 \mathrm{~min}$ (skin-to-skin), with the mean number of applications being $28.4 \pm 7.4$ per patient.

\section{Efficacy outcome analysis}

Efficacy outcomes are presented for the 70 consecutive patients who underwent HABL from July 2009 to September 2013 and had completed the 


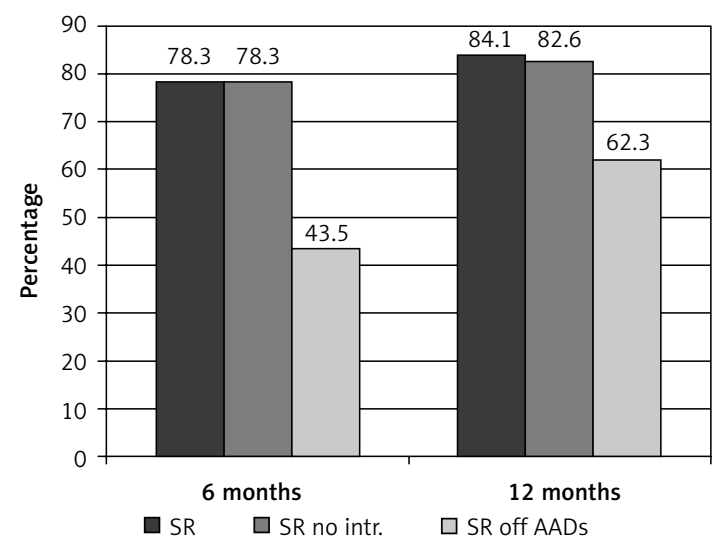

Figure 5. Efficacy outcomes of the convergent procedure

12-month post-procedure follow-up. Four of the 70 patients who were included in the efficacy analysis have not received the endocardial component of the staged HABL. The 20 patients in the remaining cohort are awaiting 12-month follow-up. Only 1 patient was lost to follow-up (deceased as described above).

At 6 months after the procedure, 78.3\% (54/69) of patients were in SR without any interventions. At 12 months after the procedure $84.1 \%$ (58/69) of patients were in SR, $82.6 \%$ were in SR without any interventions, and $62.3 \%(43 / 69)$ were in SR and off class I/III AADs. Only 1\% (1/69) of patients required a repeat ablation for atrial flutter. Figure 5 summarizes the efficacy outcomes at 6 and 12 months post-procedure time points. Transthoracic echocardiography was performed 6 and 12 months after HABL. Reverse remodeling was observed as the mean left atrial size decreased from $45.2 \pm 5.9 \mathrm{~mm}$ at baseline to $42.6 \pm 4.7 \mathrm{~mm}$ at 6 months and $42.3 \pm 6.3 \mathrm{~mm}$ at 12 months $(p<0.05)$ (Figure 6 A). The mean LVEF increased from $48.6 \pm 9.7 \%$ at baseline to 50.6 $\pm 5.6 \%$ at 6 months and $52.1 \pm 7.5 \%$ at 12 months $(p<0.01)$ (Figure 6 B).

A

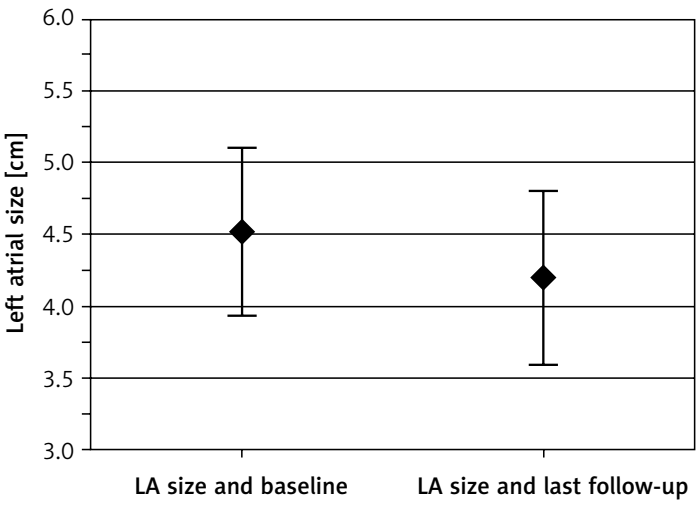

\section{Discussion}

The primary aim of this study was to assess the long-term safety, efficacy and feasibility of the staged hybrid ablation procedure for the treatment of persistent and longstanding persistent atrial fibrillation. Our previously published paper [8], based on 27 individuals with PSAF and LSPAF, provided long-term results for a small cohort of just 10 patients, and represented our early experience with the HABL. The results presented herein represent a much larger population, in which successful outcomes were noted with a low rate of complications and $1 \%$ of patients requiring a repeat ablation. Not only has the number of periprocedural complications decreased sharply, but also the effectiveness has increased. The disparity between rates at 1 year after the procedure, between sinus rhythm of $84.1 \%$ and freedom from arrhythmia and AAD of $62.3 \%$, reflects our philosophy of cautious AAD withdrawal, which in many cases of LSPAF is performed over 18-24 months rather than 1 year. In addition, patients were prescribed previously ineffective AADs; this criterion, provided there is not an increase in dosage or change in AAD regimen, is not deemed a failure in most U.S. clinical trials [12]. A similar strategy is applied to the anticoagulation regimen, as we tend not to discontinue VKA in patients with $\mathrm{CHA}_{2} \mathrm{DS}_{2}$ VASc equal to or greater than 2 despite clear 7-day Holter. Since loop monitors remain non-reimbursable, we have limited their use to patients with $\mathrm{CHA}_{2} \mathrm{DS}_{2} \mathrm{VASC}=1$ in whom VKA/ NOACs could be safely abandoned when SR was confirmed.

With time, the patient profile has also changed. Our initial experience focused on patients with a long history of AF disqualified from any percutaneous interventions. This electrophysiologists' skepticism slowly, but progressively, ceased as concise entrance-and-exit block was confirmed during CARTO mapping in consecutive patients.

B

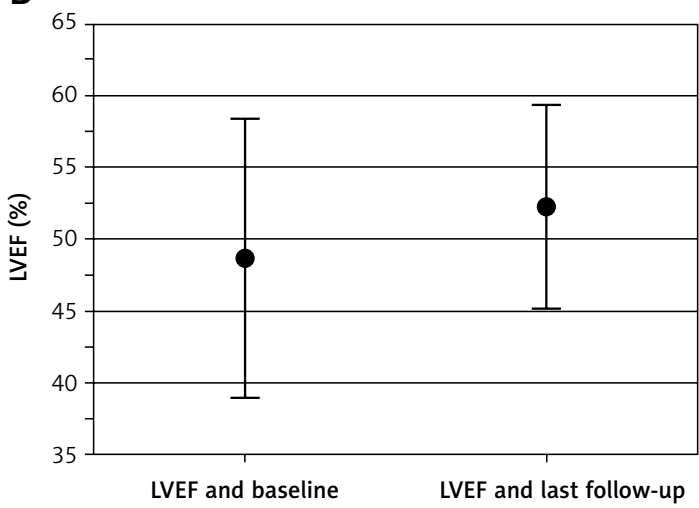

Figure 6. Change in LA size and LVEF. A - Change in LA size through 1 year F/U ( $p$-value $<0.01$ unpaired $t$-test). $\mathbf{B}-$ Change in LVEF through 1 year F/U ( $p$-value $<0.05$ unpaired $t$-test) 
Once long-term benefits had been observed, rapid referral of PSAF rather than LSPAF was noted, often as the first line of invasive treatment, as in the majority of LSPAF and PSAF with a long history of disease, the predicted efficacy of percutaneous treatment is low. Although the surgical option is more invasive, it greatly facilitates percutaneous intervention, as the EP procedure is shorter and requires less radiation exposure. Moreover, extensive ablation of the posterior left atrium, impossible to achieve with endocardial approaches, prevents esophageal injury caused by endocardial ablation. A sub-analysis revealed a higher success rate in patients in whom percutaneous pulmonary vein isolation $(\mathrm{PVI})$ was attempted previously and failed (Figures 7,8 ). With numerous publications depicting possible reasons of PVI failure, our approach remained unchanged, with aggressive ablation of the posterior LA and careful energy application around pulmonary veins. Fibrotic point-like scars were often macroscopically visible, especially in the vicinity of the posterior and inferior aspect of the left inferior pulmonary vein, not rarely accompanied by pericardial adhesions. Addition of epicardial ablation alone to the already performed endocardial lesion pattern is tempting, and may be referred to as a "reverse hybrid" approach. However, since endocardial treatment proved ineffective, and transabdominal access by itself does not allow all PVs to be isolated, we still believe that stage II (re-CARTO) is necessary, at least to demonstrate entry and exit block. Yet in our experience placement of additional endocardial applications was indispensable in every case, indicating either incompleteness of previous interventions or their superficial character. Interestingly, the number of previous endocardial procedures did not influence the success rate, indicating that one attempt, rather than repeated attempts, should be performed. This conclusion, although based on a fairly small number of patients, supports the current ESC guidelines, which indicate that surgical AF ablation should be performed after failure of percutaneous treatment. However, the guidelines fall short in as far as they do not define the number of PVI attempts prior to surgical AF ablation. Nevertheless, the results presented herein must be perceived with cautious evaluation of preoperative factors, which may significantly influence the outcome, as patients naïve to invasive treatment were, in the majority of cases, not eligible for percutaneous treatment, as the predicted success rate of PVI alone was low.

A combination of a linear, comprehensive epicardial lesion pattern with precise, CARTO-guided endocardial applications is being recognized as an efficacious treatment option for patients in whom contemporary therapies have failed [13-17]. The

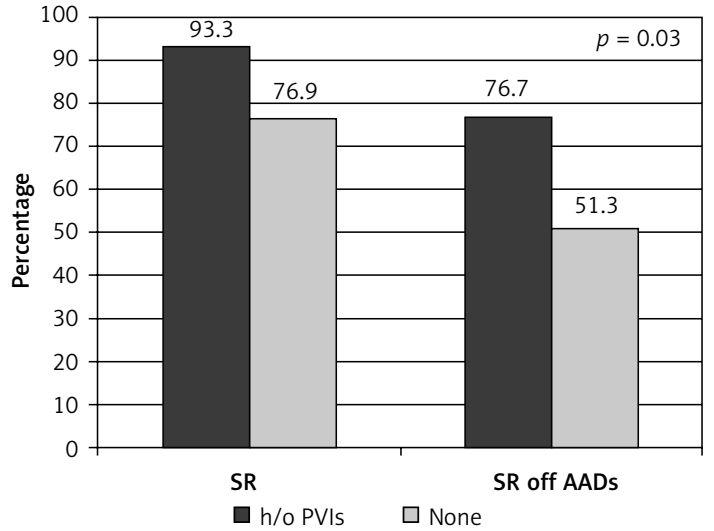

Figure 7. Sub-analysis: patients with history of endocardial treatment vs. naïve to invasive treatment

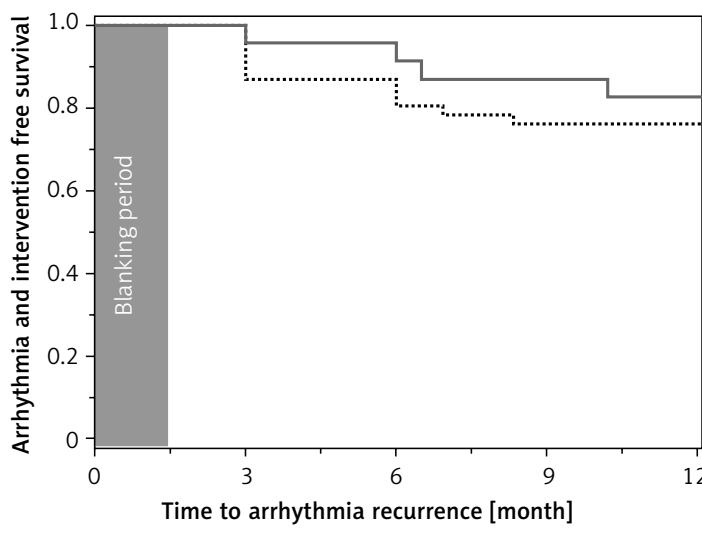

No previous ablation

$\begin{array}{ccccc}\begin{array}{l}47 \\ \text { vious ablation }\end{array} & 46 & 40 & 35 & 35 \\ 23 & 23 & 22 & 20 & 19 \\ \text {...... No previous ablation } & & \text { Previous catheter ablation }\end{array}$

Figure 8. Freedom from arrhythmia: patients with vs. without previous endocardial ablation of AF $(p=0.06)$

Convergent Procedure described here was introduced in 2009 and with over 4500 patients treated to date constitutes an important treatment option for symptomatic patients with either PSAF or LSPAF [13-15]. Success rates of maintenance of SR ranging from $76 \%$ to $100 \%$ have been reported.

It is noteworthy that the Convergent Procedure has laid the foundation and introduced the entire concept of an epicardial and endocardial, hybrid, multidisciplinary approach for the treatment of PSAF and LSPAF. It combines minimally invasive, video-assisted surgery and electrophysiology, performed with various techniques (RF or Cryo), staged or as a single setting procedure. Hybrid approaches, such as uni- or bilateral thoracoscopic PV isolation, have added endocardial ablation following surgical intervention and now offer complex, but slightly different, lesion sets [16, 18-20]. Importantly, surgical or hybrid techniques must not be looked upon as competitive, but rather 
as complementary, patient-tailored approaches. While bilateral thoracotomy allows for circular electrical isolation of both pairs of pulmonary veins, it requires chest incisions and sequential lung deflation [21]. This can be difficult to achieve in patients suffering from chronic pulmonary disease or after lung/lobe resection or inflammation, where pleural adhesions may severely limit endoscopic access [22, 23]. Transabdominal access used in the HABL is independent of lung function and anatomy, allowing for unrestricted posterior ablation and PV isolation in patients with severe COPD or other pulmonary disease. Our experience shows that successful AF ablation is possible even in patients with a history of total lung resection due to massive chest trauma. Importantly, both strategies allow for direct visualization of pulmonary veins irrespective of anatomy, and when combined with endocardial applications (either RF or Cryo) offer exceptional long-term results [24]. The LAA ligation or exclusion represents a valuable asset of the thoracoscopic approach, but, as shown here, it can also be combined safely with transabdominal access in a selected population.

While this material contributes to the growing evidence on safety and efficacy of both surgical and hybrid ablation of AF, there is a need for a prospective, randomized, controlled multicenter clinical trial (RCT) to independently assess its role in AF treatment. With thousands of patients successfully treated worldwide, surgical standalone or hybrid ablation is still considered a class IIb indication in both European and US AF treatment guidelines [25-27], limiting its use to either "highly symptomatic" patients or those in whom a percutaneous procedure (or procedures) has failed. Just by assessing our material, it is readily apparent that with a mean EHRA score of 2.6 "symptomatic", rather than "highly symptomatic" patients are treated. An analogous condition applies to patients who failed percutaneous treatment - barely $20 \%$ of the reported cohort. More importantly, a comparable patient profile is encountered in nearly all papers on surgical and/or hybrid ablation, questioning the applicability of published treatment guidelines. Yet, until there are solid data from RCTs, hybrid procedures, despite their high efficacy and excellent safety profile, will primarily be used as a last resort for patients who have failed multiple treatment modalities including percutaneous catheter ablation, or are deemed not to benefit from such treatment by the experienced Heart Team [23].

In conclusion, this 90-patient, single-center, prospective, non-randomized clinical study proves that the minimally invasive hybrid approach using transabdominal access is a safe and effective treatment option for patients diagnosed with persistent and longstanding persistent atrial fibrillation. The proce- dure efficacy was demonstrated by restoration and maintenance of sinus rhythm in $84 \%$ of patients at 1-year follow-up. Moreover, the outcomes from this single-center study indicate that restoration of sinus rhythm with no AF recurrences results in a greater improvement in the left ventricular function and reduction in the left atrial dimension.

The study suffers from its prospective, non-randomized, single treatment arm design. The results, especially in comparison to percutaneous treatment options, were based on data already published elsewhere.

\section{Conflict of interest}

Michal Zembala MD PhD served as a consultant (proctor) for nContact Inc. from January 2012 until January 2013. Other authors report no conflicts of interests. No corporate funding supported the study. The Reveal XT implantable loop monitors were purchased from funds received from the Polish Society of Cardiology/Servier Prize for the Best Research Proposal in 2011.

\section{References}

1. Chugh SS, Blackshear JL, Shen WK, Hammill SC, Gersh BJ. Epidemiology and natural history of atrial fibrillation: clinical implications. J Am Coll Cardiol 2001; 37: 371-8.

2. Benjamin EJ, Wolf PA, D'Agostino RB, Silbershatz H, Kannel WB, Levy D. Impact of atrial fibrillation on the risk of death: the Framingham Heart Study. Circulation 1998; 98: 946-52.

3. Sorgente A, Tung P, Wylie J, Josephson ME. Six year follow-up after catheter ablation of atrial fibrillation: a palliation more than a true cure. Am J Cardiol 2012; 109: 1179-86.

4. Wutzler A, Huemer M, Parwani AS, Blaschke F, Haverkamp W, Boldt LH. Contact force mapping during catheter ablation for atrial fibrillation: procedural data and one-year follow-up. Arch Med Sci 2014; 10: 266-72.

5. Je HG, Shuman DJ, Ad N. A systematic review of minimally invasive surgical treatment for atrial fibrillation: a comparison of the Cox-Maze procedure, beating-heart epicardial ablation, and the hybrid procedure on safety and efficacy. Eur I Cardiothorac Surg 2015; 48: 531-40.

6. Beukema RJ, Adiyaman A, Smit JJ, Delnoy PP, Ramdat Misier AR, Elvan A. Catheter ablation of symptomatic postoperative atrial arrhythmias after epicardial surgical disconnection of the pulmonary veins and left atrial appendage ligation in patients with atrial fibrillation. Eur J Cardiothorac Surg 2016; 49: 265-71.

7. European Heart Rhythm Association (EHRA); European Cardiac Arrhythmia Society (ECAS); American College of Cardiology (ACC); American Heart Association (AHA); Society of Thoracic Surgeons (STS), Calkins H, Brugada J, Packer DL, et al. HRS/EHRA/ECAS expert Consensus Statement on catheter and surgical ablation of atrial fibrillation: recommendations for personnel, policy, procedures and follow-up. A report of the Heart Rhythm Society (HRS) Task Force on catheter and surgical ablation of atrial fibrillation. Heart Rhythm 2007; 4: 816-61. 
8. Zembala M, Filipiak K, Kowalski O, et al. Minimally invasive hybrid ablation procedure for the treatment of persistent atrial fibrillation: one year results. Kardiol Pol 2012; 70: 819-28.

9. Kalarus Z, Zembala M, Kowalski O, et al. [Minimally invasive, hybrid ablation in a patient with persistent atrial fibrillation - early experience]. Kardiol Pol 2009; 67: 1057-62.

10. Gage BF, Waterman AD, Shannon W, Boechler M, Rich MW, Radford MJ. Validation of clinical classification schemes for predicting stroke: results from the National Registry of Atrial Fibrillation. JAMA 2001; 285: 2864-70.

11. Olesen JB, Lip GY, Hansen ML, et al. Validation of risk stratification schemes for predicting stroke and thromboembolism in patients with atrial fibrillation: nationwide cohort study. BMJ 2011; 342: d124.

12. Packer DL, Kowal RC, Wheelan KR, et al. Cryoballoon ablation of pulmonary veins for paroxysmal atrial fibrillation: first results of the North American Arctic Front (STOP AF) pivotal trial. J Am Coll Cardiol 2013; 61: 1713-23.

13. Gersak B, Zembala MO, Muller D, et al. European experience of the convergent atrial fibrillation procedure: multicenter outcomes in consecutive patients. J Thor Cardiovasc Surg 2014; 147: 1411-6.

14. Gersak B, Pernat A, Robic B, Sinkovec M. Low rate of atrial fibrillation recurrence verified by implantable loop recorder monitoring following a convergent epicardial and endocardial ablation of atrial fibrillation. J Cardiovasc Electrophysiol 2012; 23: 1059-66.

15. Kiser AC, Landers M, Horton R, Hume A, Natale A, Gersak $B$. The convergent procedure: a multidisciplinary atrial fibrillation treatment. Heart Surg Forum 2010; 13 : E317-21.

16. Pison L, Gelsomino S, Luca F, et al. Effectiveness and safety of simultaneous hybrid thoracoscopic and endocardial catheter ablation of lone atrial fibrillation. Ann Cardiothorac Surg 2014; 3: 38-44.

17. Sarkozy A, La Meir M, Rodriguez-Manero M, Brugada P. Endocardial completion of epicardial linear ablation during hybrid thoracoscopic and catheter ablation of persistent atrial fibrillation. Rev Esp Cardiol 2013; 66: 822-3.

18. Bisleri G, Curnis A, Cheema FH, Muneretto C. Sequential hybrid ablation for persistent atrial fibrillation. Ann Thorac Surg 2012; 94: 689-90; author reply 90.

19. Muneretto C, Bisleri G, Bontempi L, Curnis A. Durable staged hybrid ablation with thoracoscopic and percutaneous approach for treatment of long-standing atrial fibrillation: a 30-month assessment with continuous monitoring. J Thor Cardiovasc Surg 2012; 144: 1460-5; discussion 65

20. Pison L, La Meir M, van Opstal J, Blaauw Y, Maessen J, Crijns HJ. Hybrid thoracoscopic surgical and transvenous catheter ablation of atrial fibrillation. J Am Coll Cardiol 2012; 60: 54-61.

21. Boersma LV, Castella M, van Boven W, et al. Atrial fibrillation catheter ablation versus surgical ablation treatment (FAST): a 2-Center Randomized Clinical Trial. Circulation 2012; 125: 23-30.

22. La Meir M. Re: Thoracoscopic surgical ablation versus catheter ablation for atrial fibrillation. Eur J Cardiothorac Surg 2016; 49: 1052-3.

23. Phan K, Phan S, Thiagalingam A, Medi C, Yan TD. Thoracoscopic surgical ablation versus catheter ablation for atrial fibrillation. Eur J Cardiothorac Surg 2016; 49 1044-51.
24. Gelsomino S, Van Breugel HN, Pison L, et al. Hybrid thoracoscopic and transvenous catheter ablation of atrial fibrillation. Eur J Cardiothorac Surg 2014; 45: 401-7.

25. Calkins H, Kuck KH, Cappato R, et al. 2012 HRS/EHRA/ ECAS expert consensus statement on catheter and surgical ablation of atrial fibrillation: recommendations for patient selection, procedural techniques, patient management and follow-up, definitions, endpoints, and research trial design: a report of the Heart Rhythm Society (HRS) Task Force on Catheter and Surgical Ablation of Atrial Fibrillation. Developed in partnership with the European Heart Rhythm Association (EHRA), a registered branch of the European Society of Cardiology (ESC) and the European Cardiac Arrhythmia Society (ECAS); and in collaboration with the American College of Cardiology (ACC), American Heart Association (AHA), the Asia Pacific Heart Rhythm Society (APHRS), and the Society of Thoracic Surgeons (STS). Endorsed by the governing bodies of the American College of Cardiology Foundation, the American Heart Association, the European Cardiac Arrhythmia Society, the European Heart Rhythm Association, the Society of Thoracic Surgeons, the Asia Pacific Heart Rhythm Society, and the Heart Rhythm Society. Heart Rhythm 2012; 9: 632-96 e21.

26. European Heart Rhythm Association; European Association for Cardio-Thoracic Surgery, Camm AJ, Kirchhof P, Lip GY, et al. Guidelines for the management of atrial fibrillation: the Task Force for the Management of Atrial Fibrillation of the European Society of Cardiology (ESC). Europace 2010; 12: 1360-420.

27. Fuster V, Ryden LE, Cannom DS, et al. 2011 ACCF/AHA/ HRS focused updates incorporated into the ACC/AHA/ ESC 2006 Guidelines for the management of patients with atrial fibrillation: a report of the American College of Cardiology Foundation/American Heart Association Task Force on Practice Guidelines developed in partnership with the European Society of Cardiology and in collaboration with the European Heart Rhythm Association and the Heart Rhythm Society. J Am Coll Cardiol 2011; 57: e101-98. 